\title{
Imag(in)ing the Nano-scale: Introduction
}

\author{
Rasmus Tore Slaattelid • Fern Wickson
}

Received: 1 July 2011 / Accepted: 13 July 2011 /Published online: 27 July 2011

(C) Springer Science+Business Media B.V. 2011

This special issue of Nanoethics is the result of four workshops. The first one at the 2009 S.NET conference in Seattle, the second at the Maison des Sciences de L'homme in Paris later the same year, the third at the 2010 S.NET Conference in Darmstadt, and finally a fourth workshop at the University of Bergen in January 2011. The aim of the meetings was, apart from the pure pleasure of stimulating intercourses over papers, luncheons and dinners, to explore visualisations of the nano-scale present across science, public media and art, from the perspective of the humanities. The papers in this issue present a range of perspectives on nano visualisations, drawing upon models of interpretation from fields as diverse as the iconology of St. John of Damascus, to cultural studies of play and gaming in modern life.

It is a truism that images are central to nano-scale sciences and technologies (NST). In a certain sense, these practices "live[s] from the production and

\section{R. T. Slaattelid}

Centre for the Study of the Sciences and the Humanities, University of Bergen,

$\mathrm{Pb}$. 7805, 5020 Bergen, Norway

F. Wickson

GenØk Centre for Biosafety,

PB 6418, 9294 Tromsø, Norway

R. T. Slaattelid $(\bowtie)$

Senter for vitskapsteori, Universitetet i Bergen, $\mathrm{Pb}$. 7805, 5020 Bergen, Norway

e-mail: hfirs@uib.no mediation of images' (Baird et al. 2004). Images play an indispensable role in research as heuristic tools and empirical models, and as documentation of research results. They are instrumental in communicating scientific development to the scientific community, as well as to the broader public, and they are crucial in shaping the collective imaginaries that will eventually constitute the public perception of NST as well as future frameworks for policy. These all represent good reasons to put effort into increasing our understanding of how visualisations of the nanoscale work across all these contexts.

\section{Existing Humanities Research on Nano Images}

While it can be argued that humanities research on nano-scale sciences and technologies is limited in comparison to the work being done within the social sciences, an increasing amount of humanities based research is specifically directed towards visualisations of the nano-scale. Some of this research takes an historical perspective on scientific visualisations, particularly focused on the development of NST imaging instrumentation, such as the scanning tunnelling microscope (STM) (e.g. $[1,5,6])$, or on the design of images from such instrumentation (e.g. [4]). By describing the instrumentation and the shifting design of images generated by such instruments, this research has been (indirectly) able to show the influence of cultural values and visions on their production and acceptance. For example, Baird \& 
Shew [1] argue that the development of the STM and its increasing importance as a tool in NST has been influenced by a 'post academic' social and cultural context (see [7,17]) in which science is much more closely tied to commercial activity. Hennig [4] argues that images generated from STM data have gradually shifted in their design so that the role of the instrument is now backgrounded, and the images themselves have been made compatible with existing cultural expectations (such as what atoms should look like) and hopes for the future (such as a human ability to control matter on this scale). Hessenbruch [5] also discusses the role of STMs in supporting and coproducing the promise and hype associated with the idea of control of matter on the atomic scale.

Research on images of the nano-scale from a philosophical perspective has also been pursued (e.g. $[3,9,11-13]$,$) . This research has, in addition to$ discussing epistemological issues, also articulated ways in which narratives, metaphors and values influence and are influenced by NST imagery. Nordmann [9] for example discusses how nano images draw on traditional and familiar concepts and metaphors (such the conquest of outer space) and redeploy these in new contexts (e.g. nano as the conquest of inner space). Pitt [12] focuses on the very concept of 'seeing' and how this concept requires revision when STMs are claimed to allow us to 'see' atoms. Daston and Galison [3] focus on the haptic 'sight' of scanning probe microscopy where the production of the phenomenon to be recorded and the actual imaging in some cases can be seen as one and the same process.

Other strands of philosophical research on nano imagery have emphasised ethical dimensions of the relationship between object and image. Pitt [13] argues that presenting scientific visualisations of the nano-scale as 'images' rather than 'imaginings' fails to 'tell the public the truth about what we can and cannot know' [13]. Nordmann [10] also highlights the ethical problems of the revealing/concealing dimension of the imag(in)ing process and calls specifically for philosophy of science to take up a 'new task'- to critically engage with, deliberate upon, and 'do battle against the models and images of technoscience by making explicit their implicit claims to represent and by showing where these claims run into difficulty' [10]. Cultural anthropologist Chris Toumey also emphasises the importance of ethical questions raised by the manipulations involved in scientific imag(in) ings, such as the use of artificial colours and shading [15]. For Toumey [15], an ethical approach to the presentation of these imag(in)ings requires the addition of a temporal dimension (showing how the image has been constructed through time), a colour perspective (to demonstrate the way colours have been artificially added and how this could be done differently) and a tactile perspective (to convey the sense of 'feeling' that is crucial to the operation of STMs and AFMs).

Other important contributions in the field have come from artists [14] and linguists [8]. Robinson [14] picks up on the aforementioned topic of how NST images are changing what we mean by the concept of 'seeing' and specifically argues for art as an alternative form and method of discovery that has traditionally had a more direct interest in and awareness of its relations to the culture in which it is embedded. Robinson [14] suggests that while the art world has remained largely unaware of the development of NST, the arts are in a perfect position to highlight and evaluate the cultural interactions of this development. He also specifically suggests that creative interdisciplinary work in this field 'offers the opportunity for inventive visual discovery' [14]. Nerlich [8] investigates a collection of what Robinson classifies as 'fantasy' images, specifically those depicting nano-sized robots (or nanobots), and asks what cultural metaphors, visions and expectations these images employ and create. Nerlich argues that the nanobot fantasy images that have been produced as a way of visualising the nano-scale serve to normalise this, as yet undeveloped, technology and encourage public acceptance and enthusiasm for NST as a whole.

\section{Imaging? Imagining? Imag(in)ing}

The publication of visualisations of the nano-scale is proliferating in a wide variety of media. Nano images have not only become part of the scientific, but also of the general visual culture. A recurrent question in discussions of nano-scale visualisations is how we should make sense of the fact that they visualise something that is strictly speaking not accessible to vision, or any other mode of sense-perception. What we look at cannot be seen. This paradoxical feature 
has offered opportunity for renewed reflection upon the nature and limits of representation, where nano visualisations seem to resist, to a greater degree than other scientific visualisations, the traditional accounts of the representational relationship between object and image. In order to deal with this puzzling situation, different strategies are at hand in the repertoire of philosophical therapeutics. One is to insist that we stop talking about seeing atoms, and about visualisations of the nano-scale as 'images'. 'Seeing' and 'image' suggest representation, and consequently, we should in the name of truthfulness, refrain from using these suggestive terms. Instead we should speak of them as 'heuristic imaginings' and also take the trouble to explain the difference to the public [13]. Whether or not this will solve the puzzle of representation of the nano-scale depends upon your epistemological beliefs as well as upon linguistic conventions. Probably we will not see captions anytime soon stating: 'Fig. 1 is my heuristic imagining of two iron atoms on a copper plate', simply because this is not the way researchers speak about their visualisations. Such a strategy of "linguistic purification" has met with criticism from practitioners. Birkeland and Strand [2] argue that nano images should not be judged by their ability to represent reality, but by their ability to convey relevant information to researchers in given contexts. Instead of linguistic purification, they advocate linguistic extension to cover the new use of the term, along with increased efforts in enhancing public understanding of nano images as research tools. The term image is perfectly legitimate as long as the extension of the term is adjusted to fit the actual practice. [2]

How can we develop our understanding of the production, use, dissemination and effects of nano images, once we have realized that they are not representations of reality in any straightforward sense of the word? How do we proceed, after critical epistemology has rightly pointed out the inadequacies of a traditional representationalist account of nano visualisations? [16] Not surprisingly NST moves forward without worrying about the aporias of representation, and imaging technologies remain a central driver in their development. The visualisations or imag(in)ings generated fulfil a variety of functions and are interwoven with objects, instruments, theories, people, symbolic systems, other images, practi- ces and social institutions which all contribute to their constitution. This is the point from which the present collection of articles takes off, in different directions, but with one common goal: to reach an understanding of nano visualisations beyond the paradoxes of representation. Ceci n'est pas un atome? What then?

\section{The Papers in This Volume}

CATHERINE ALLAMEL-RAFFIN uses a microsemiotic framework to analyze in detail how an image acquires meaning in the course of conversations between researcher(s) and a microscopist around a SEM as a sample is being examined. Her analysis focuses on how the meaning of the visual representation emerges, and how disagreement about meaning is rooted in differences in knowledge and expectations, leading in turn to differences in emphasis on semiotic elements. Allamel-Raffin argues that the visual representation of the data collected by the instrument is modified as a result of this meaning-generating exchange.

VINCENT BONTEMS offers a phenomenotechnical (Bachelard) analysis of the creation of a nano image, followed by a discussion of its life-cycle (Simondon) in the scientific field. He ends with reflections about the "afterlife" of nano-images, that is, when they leave the scientific domain and enter into association with other types of images in the general culture and become social signs. The conclusion of the paper offers a strategy for helping the layperson accommodate to such images.

MARTIN RUIVENKAMP and ARIE RIP take up the distinction between image and imagining, in focusing on the interconnections, or entanglements between the two. The entanglements are not viewed as problems to be solved, but as de facto features of nano visualisations. Three arenas of entanglement are identified and discussed-production practices and use; future and present; nanoscience and art. The argument of the paper is that imagining is profoundly entangled in technoscientific data-based imaging practices. Also, the relationship between present achievements, and the achievements the future might hold for nanotechnology, is frequently entangled, as is the relationship between data-based imaging and socalled "artist's impressions". The authors conclude by suggesting that entanglements between imaginings and images are sometimes stabilized, but for the most 
part they are dynamic and shifting, depending upon the contexts to which they are subjected.

ALEXEI GRINBAUM takes the Byzantine iconology of St. John of Damascus as his point of departure for exploring the ways we understand nano visualisations. This proves to be a fruitful analogy for discussing the nature of the growing divide between those who possess the knowledge to appropriately understand nanotechnological images, and those who must resort to scientifically inadequate resources of knowledge in order to make sense of them, or simply venerate them as nanotechnological icons. Grinbaum suggests that we use the Byzantine experience with divine images to learn the importance of enhancing scientific visual literacy when it comes to nanotechnological icons.

SACHA LOEVE, in a philosophically informed study, points to a way out of the paradoxes of representation with respect to nano images. Drawing upon intellectual resources in the history of philosophy, he identifies strands of thinking about images that can bypass the distinction between images and imaginings suggested by traditional representationalism. Based on some significant differences between far-field and near-field microscopies, the author suggests that instead of thinking about STM images as representations of an object, they should be thought of as resulting from a dialogue between the object and the probe, an idea conceptualised by the neologism 'imaginaction'. One implication of such a reconsideration of representation is that the so-called "artist's impressions", in order to take their political role seriously, should strive to give expression to the "collapse of representation", rather than concealing it.

COLIN MILBURN takes seriously the playfulness of nanotechnology in a paper that tells the story of two nano-artifacts and their adventures in nanotechnology and nanoculture; the nanocar and the nano soccerball. The stories reveal a serious aspect of what sometimes seems to be a puerile obsession with toys in nano imaging. Without reducing it to an insignificant by-product of serious nano research, Milburn, on the contrary shows how this tendency towards play and jokes can be seen as intrinsic to technoscientific practice.

ASTRID SCHWARZ and ALFRED NORDMANN invite us to go beyond the question of representation by going for a walk - a stroll- through the 'image- scapes' of nanotechnology. For an adequate understanding of visualisations of the nano-scale, we should not solely rely on the criteria of veracity and representation, since this would result in a perspective that misses the technoscientific nature of these images. The images should rather be understood from the perspective of engineering, which is based on the assumption that nanocosm can be controlled and transformed into a something familiar, adaptable, and useful. Adopting the methodology of Spaziergangswissenschaft (strollology) the authors focus on how certain nano images (imagescapes) invite the beholder into its world, affording a certain view of this world in terms of what can be seen, and how.

As imagescapes may invite the beholder into a nanoworld, the papers in this collection invite the readers of Nanoethics to engage in a critical dialogue on visualisations of the nano-scale, and it is our hope that the readers will have as much fun reading them as we had in discussing and writing them.

Finally, we would like to thank all the participants at all four workshops for contributing to the revisions of the manuscripts at various stages, and Judith Larsen especially, for her assistance in the editorial process. The workshops leading up to this special issue, as well as the work on the issue itself, was supported at various stages by the Research Council of Norway, the University of Bergen under the FRIFORSK funding scheme, the Centre franconorvégien en sciences sociales et humanies, and the Fondation Maison des sciences de l'homme.

\section{References}

1. Baird D, Shew A (2004) Probing the history of scanning tunneling microscopy. In: Baird D, Nordmann A, Schummer J (eds) Discovering the nanoscale. Ios Press, Amsterdam, pp 145-156

2. Birkeland T, Strand R (2009) How to understand nano images. Techné: Research in Philosophy and Technology 13 (3):182-189

3. Daston L, Galison P (2007) Objectivity. Zone Books, New York

4. Hennig J (2006) Changes in the design of scanning tunneling microscopic images from 1980 to 1990. In: Schummer J, Baird D (eds) Nanotechnology challenges: implications for philosophy, ethics and society. World Scientific Publishing, Singapore, pp 143-163

5. Hessenbruch A (2004) Nanotechnology and the negotiation of novelty. In: Baird D, Nordmann A, Schummer J (eds) 
Discovering the nanoscale. Ios Press, Amsterdam, pp 135144

6. Mody CC (2004) How probe microscopists became nanotechnologists. In: Baird D, Nordmann A, Schummer J (eds) Discovering the nanoscale. Ios Press, Amsterdam, pp 119134

7. Moriarty P (2008) Reclaiming academia from postacademia. Nat Nanotechnol 3:60-62

8. Nerlich B (2008) Powered by imagination: nanobots in the science photo library. Science as Culture 17(32):269-292

9. Nordmann A (2004) Nanotechnology's worldview: new space for old cosmologies. IEEE Technology and Society Magazine 23(4):48-54

10. Nordmann A (2006) Collapse of distance: epistemic strategies of science and technoscience. Danish Yearbook of Philosophy 41:7-34

11. Nordmann A (2008) Philosophy of nanotechnoscience. In: Schmid G, Krug H, Waser R, Vogel V, Fuchs H, Grätzel M, Kalyanasundaram K, Chi L (eds) Nanotechnology, vol. 1:
G. Schmid (ed.) Principles and fundamentals. Wiley, Weinheim, pp 217-244.

12. Pitt JC (2004) The epistemology of the very small. In: Baird D, Nordmann A, Schummer J (eds) Discovering the nanoscale. Ios Press, Amsterdam, pp 157-164

13. Pitt J (2006) When is an image not an image? In: Schummer J, Baird D (eds) Nanotechnology challenges: implications for philosophy, ethics and society. World Scientific Publishing, Singapore, pp 131-141

14. Robinson C (2004) Images in nanoscience/technology. In: Baird D, Nordmann A, Schummer J (eds) Discovering the nanoscale. Ios Press, Amsterdam, pp 165-172

15. Toumey C (2007) Cubism at the nanoscale. Nat Nanotechnol 2:587-589

16. Toumey C (2010) Images and icons. Nat Nanotechnol 5:34

17. Ziman J (1998) Why must scientists become more ethically sensitive than they used to be? Science 282(5395):18131814 